\title{
Remaining Useful Life Estimation Using an Inverse Gaussian Degradation Model
}

\author{
Donghui Pan ${ }^{\mathrm{a}}$, Jia-Bao Liu ${ }^{\mathrm{b}}$, Jinde $\mathrm{CaO}^{\mathrm{c}, \mathrm{d}, *}$ \\ ${ }^{a}$ School of Mathematical Sciences, Anhui University, Hefei 230601, P. R. China \\ ${ }^{b}$ Department of Public Courses, Anhui Xinhua University, Hefei 230088, China \\ ${ }^{c}$ Research Center for Complex Systems and Network Science, Department of Mathematics, Southeast University, Nanjing 210096, P. R. China \\ ${ }^{d}$ Department of Mathematics, Faculty of Science, King Abdulazez University, Jeddah 21589, Saudi Arabia
}

\begin{abstract}
The use of degradation data to estimate the remaining useful life (RUL) has gained great attention with the widespread use of prognostics and health management on safety critical systems. Accurate RUL estimation can prevent system failure and reduce the running risks since the efficient maintenance service could be scheduled in advance. In this paper, we present a degradation modeling and RUL estimation approach by using available degradation data for a deteriorating system. An inverse Gaussian process with the random effect is firstly used to characterize the degradation process of the system. Expectation maximization algorithm is then adopted to estimate the model parameters, and the random parameters in the degradation model are updated by Bayesian method, which makes the estimated RUL able to be real-time updated in terms of the fresh degradation data. Our proposed method can capture the latest condition of the system by means of updating degradation data continuously, and obtain the explicit expression of RUL distribution. Finally, a numerical example and a practical case study are provided to show that the presented approach can effectively model degradation process for the individual system and obtain better results for RUL estimation.
\end{abstract}

(c) 2011 Published by Elsevier Ltd.

Keywords: Degradation modeling, Inverse Gausian process, Random effect, Remaining useful life

\section{Introduction}

The great majority of engineering systems inevitably deteriorate along with usage time as a result of multi-source stresses resulting from either the external environments or internal degradation. With the rapid development of sensor and information technology, degradation processes that arise during the life cycle of engineering systems can be observed relatively easily through condition monitoring technologies. The observed condition-based data are usually known as degradation data and are closely related to the underlying physical degradation process [29, 35]. Several examples of degradation data involve fatigue crack growth data [34], light intensity data [30], capacity data of lithiumion batteries $[8,13]$, vibration data $[6,9,10]$, drift data of the gyro $[22,23]$, wear data of hard disk drives [32] and so forth. To guarantee the safe, reliable, and effective operation of an engineering system, prognostics and health management (PHM) has received considerable attention in academia and industry in the past decades. The fundamental

\footnotetext{
${ }^{*}$ Corresponding author

Email addresses: dhpan@ahu.edu.cn (Donghui Pan), liujiabaoad@163.com (Jia-Bao Liu), jdcao@seu.edu.cn (Jinde Cao)
} 
objective of PHM is to enable system health monitoring applications that ensure the system operation within the scope of design limits. PHM includes the following two parts: prognostics and health management. Prognostics is regarded as the key of PHM because it is generally used as the basis of health management. In reality, prognostics largely focuses on estimating the remaining useful life (RUL) of the interested system by using the failure mechanism or the available degradation data. Therefore, RUL estimation plays a key role in system PHM, which can offer adequate lead-time for the maintainer to implement the essential maintenance actions ahead of failure.

The existing RUL estimation approaches can be broadly categorized into physics-based approaches, data-driven approaches and their hybrid [7]. The physics-based approaches usually needs explicit mathematical model to quantitatively characterize the behavior of a degrading system to estimate the RUL [1]. However, it is typically difficult or even impossible to understand the failure mechanism of a degrading system, especially for the complicated or largescale systems under dynamic operating environment, which prevent practitioners to develop accurate mathematical models for RUL estimation. In addition, the degradation data can be obtained easier and easier by using condition monitoring technologies. For these reasons, data-driven approaches have become the mainstream choice in the RUL estimation field without physical understanding about the degradation process of the system.

In the existing data-driven RUL estimation approaches, the stochastic process based approaches have been well accepted as the most effective methods [12, 24]. Two kinds of the most familiar stochastic process are Wiener and Gamma processes [25, 21, 28, 33], which have been well researched due to their mathematical advantages and physical interpretations. Although these two classes of stochastic processes have been widely used in degradation modeling, they cannot fit degradation data well in some practical applications. Wang and $\mathrm{Xu}$ [30] demonstrated that both Wiener and Gamma processes fit the GaAs Laser data poorly, and suggested inverse Gaussian (IG) process as a fine alternative for degradation modeling. Qin et al. [18] has showed the flexibility of IG process for degradation modeling of energy pipelines. Ye and Chen [31] inquired deeply into the physical meanings of IG process for degradation modeling and proved that IG process is the limit of a compound Poisson process. Their research has also provided three IG degradation models with random effects. Peng [15] proposed an inverse normal-gamma mixture of an IG process model for degradation data. Peng et al. [16] introduced a Bayesian analysis of IG process models for degradation modeling and inference. Liu et al. [11] developed a reliability modeling method for multiple degradation process based on an IG process and copulas. Although the IG process is applied to degradation modeling, it was scarcely used in the RUL estimation. The above existing IG process based degradation models only pay attention to estimate population-related reliability characteristics for a population of identical systems from the same batch, such as timeto-failure distributions. In such cases, the estimated life is actually the mean time to failure for a kind of systems, which can be useful at the design or testing stage before the real system is put into use. However, it will be more desirable to perform the online RUL estimation using the real-time observed degradation data for a specific system in service. In comparison with existing approaches, our primary objective of this paper is to adaptively estimate the RUL of a system in service by using the partial observation data of its degradation process. The online RUL estimations for the system in service are usually utilized for support decision-related applications, such as spare parts inventory, replacement and maintenance scheduling and so on.

Motivated by this practical need, this paper is to develop an adaptive RUL estimation approach based on an IG process with the random effect, where the random effect is utilized to characterize the unit-to-unit heterogeneities for the degrading systems from the same batch. To be specifical, the random parameter can be real-time updated by the Bayesian rule using the available degradation data of the interested system in use, and the probability density function (PDF) of the RUL can be dynamically updated adaptively to the real-time health conditions, where the uncertainty of the random parameter is also incorporated. The main contributions of this paper are summarized as follows. Firstly, an adaptively RUL estimation approach is proposed using an IG process with the random effect for the degrading system in service. With the aid of the Bayesian updating mechanism, the PDF of the estimated RUL can be dynamically updated on the basis of the arrivals of new degradation data from the interested system. Secondly, the uncertainty associated with the random effect characterizing the unit-to-unit heterogeneities is incorporated into the real-time estimated RUL distribution, which plays an important role in the following high-level decision-making. Eventually, our approach can obtain an explicit expression of the RUL distribution, and there also exists the moment of the obtained RUL distribution. Additionally, the parameter updates of every iteration of the EM algorithm have explicit expression, which makes each iteration only require a single calculation.

The remainder of this paper is organized as follows. Section 2 presents the degradation modeling and RUL estimation approach, while the statistical Inference procedure is specified in Section 3. Section 4 provides a simulated 
example and a practical case study to demonstrate the proposed degradation modeling and RUL estimation approach. Section 5 concludes this paper.

\section{Degradation modeling and RUL estimation}

\subsection{Degradation modeling}

In practice, the performance of numerous systems or components degrades over time, which can be modeled by a stochastic process. In our research, we will employ the IG process to represent monotonic degradation process. An IG process model is defined for a degradation process $\{Y(t), t \geq 0\}$ satisfying the following properties.

- $Y(0)=0$ with probability one;

- $Y(t)$ has independent increments on nonoverlapping intervals, i.e., $Y\left(t_{2}\right)-Y\left(t_{1}\right)$ and $Y\left(t_{4}\right)-Y\left(t_{3}\right)$ are independent for $\forall 0 \leq t_{1}<t_{2} \leq t_{3}<t_{4}$

- each increment follows an IG distribution, i.e., $Y(t+\Delta t)-Y(t) \sim I G\left(\beta \Delta \Lambda, \eta(\Delta \Lambda)^{2}\right)$ for $\forall \Delta t>0$;

where $\Delta \Lambda=\Lambda(t+\Delta t)-\Lambda(t), \Lambda(t)$ is a given, monotone increasing function with $\Lambda(0)=0$, and the PDF of $I G\left(\beta \Lambda(t), \Lambda(t)^{2}\right)$ is defined by

$$
f_{I G}(y \mid \beta, \eta)=\left(\frac{\eta \Lambda(t)^{2}}{2 \pi y^{3}}\right)^{1 / 2} \exp \left[-\frac{\eta}{2 y}\left(\frac{y}{\beta}-\Lambda(t)\right)^{2}\right]
$$

The IG process $Y(t)$ then has mean $\beta \Lambda(t)$ and variance $\beta^{3} \Lambda(t) / \eta$. The parameter $\beta$ denotes the degradation rate, and the parameter $\eta$ has no definite physical meaning. The shape function $\Lambda(t)$ denotes the measurement of a physical progress of degradation, such as corrosion, oxidation, and fatigue growth, which relies heavily on the certain failure mechanism that is dominated by a specific application. On the condition that $\Lambda(t)$ is a linear function, the IG process represents a stationary process. However, the degradation increment generally relies on both $t$ and $\Delta t$, which turns out to be a non-stationary process. In our research, we consider a IG-process-based degradation model with a linear shape function (i.e. $\Lambda(t)=t$ ), since the linear degradation model is generally used to model the degradation processes where the degradation rate increases linearly over time $[3,4,5,17]$.

In practice, each system or component can undergo various operating conditions, which causes the degradation process of diverse systems to manifest distinct degradation rates. For this reason, it is certainly preferable to incorporate unit-to-unit heterogeneity in the degradation model. Therefore, we regard the parameter $\beta$ to be a random parameter describing unit-to-unit variability, and $\eta$ to be a deterministic parameter. In order to facilitate the subsequent modelling and mathematical simplification, we assume the prior distribution of $1 / \beta$ follows the normal distribution, i.e. $1 / \beta \sim N\left(\mu_{\beta}, 1 / \sigma_{\beta}^{2}\right)$, and is statistically independent of $\eta$. The ideas of random effects and Gaussian assumptions are widely used in degradation modeling literature. Other distributions may also act as the prior distribution of $1 / \beta$, but it might need to adopt the Markov chain Monte Carlo method to estimate the posterior distribution of $1 / \beta$.

\subsection{RUL estimation with the random effect}

The system is deemed to be failed when the degradation path first reaches a predefined threshold level in many engineering applications [25]. For this reason, we adopt the concept of the first hitting time (FHT) of the IG process $\{Y(t), t \geq 0\}$ to define the life, and then derive the RUL. The distribution of the FHT plays a key role in RUL estimation and in determining the optimal maintenance strategies. Without loss of generality, we suppose that the degradation process crosses the predefined threshold $\omega$, the system is considered as a failure. On the basis of the concept of the FHT, the life $T$ is defined as $T=\inf \{t \mid Y(t) \geq \omega\}$. As a result of the monotonicity property of the IG process, the cumulative distribution function $(\mathrm{CDF})$ of $T$ can be represented as

$$
\begin{aligned}
F_{T \mid \beta}(t \mid \beta)=P(T<t)=P(Y(t)>\omega) & =1-F_{I G}\left(\omega \mid \beta t, \eta t^{2}\right) \\
& =\Phi\left[\underset{3}{\Phi}\left[\sqrt{\frac{\eta}{\omega}}\left(t-\frac{\omega}{\beta}\right)\right]-\exp \left(\frac{2 \eta t}{\beta}\right) \Phi\left[-\sqrt{\frac{\eta}{\omega}}\left(t+\frac{\omega}{\beta}\right)\right],\right.
\end{aligned}
$$


where $F_{I G}(\cdot)$ denotes the CDF of the IG distribution. By taking the derivative with respect to $t$, the PDF of $T$ can be easily obtained as

$$
f_{T \mid \beta}(t \mid \beta)=2 \sqrt{\frac{\eta}{\omega}} \phi\left[\sqrt{\frac{\eta}{\omega}}\left(t-\frac{\omega}{\beta}\right)\right]-\frac{2 \eta}{\beta} \exp \left[\frac{2 \eta t}{\beta}\right] \Phi\left[-\sqrt{\frac{\eta}{\omega}}\left(t+\frac{\omega}{\beta}\right)\right],
$$

where $\phi(\cdot)$ and $\Phi(\cdot)$ are the PDF and CDF of the standard normal distribution respectively. In addition, when the parameter $\beta$ is fixed, according to the equation (2), the mean of the life (i.e. mean-time-to-failure) can be easily formulated by:

$$
E(T \mid \beta)=\int_{0}^{\infty} t f_{T \mid \beta}(t \mid \beta) d t=\left(\frac{\omega}{\beta}+\frac{\beta}{\eta}\right) \Phi\left(\frac{\sqrt{\eta \omega}}{\beta}\right)+\sqrt{\frac{\omega}{\eta}} \phi\left(\frac{\sqrt{\eta \omega}}{\beta}\right)-\frac{\beta}{2 \eta} .
$$

It should be noted that the obtained CDF and PDF of the life $T$ is still conditional on the random parameter $\beta$. In view of the random nature of $\beta$, we can derive the unconditional CDF and PDF of the life $T$ using the law of total probability as follows:

$$
F_{T}(t)=\int_{\Omega} F_{T \mid \beta}(t \mid \beta) p(\beta) d \beta=E_{\beta}\left[F_{T \mid \beta}(t \mid \beta)\right],
$$

and

$$
f_{T}(t)=\int_{\Omega} f_{T \mid \beta}(t \mid \beta) p(\beta) d \beta=E_{\beta}\left[f_{T \mid \beta}(t \mid \beta)\right],
$$

where $p(\beta)$ and $\Omega$ represent the PDF and parameter space of the random parameter $\beta$ respectively, $E_{\beta}[\cdot]$ is the expectation operator in relation to $\beta$. To explicitly calculate the integrals above, we firstly provide the following lemma, which can simplify the derivation process for the life distribution.

Lemma 1. If $Z \sim N\left(\mu, \sigma^{2}\right)$ and $c, \gamma, \lambda \in \mathbf{R}$, then the following holds:

$$
E_{Z}[\exp (\lambda Z) \Phi(c+\gamma Z)]=\exp \left(\lambda \mu+\frac{\lambda^{2} \sigma^{2}}{2}\right) \Phi\left(\frac{c+\gamma \mu+\lambda \gamma \sigma^{2}}{\sqrt{1+\gamma^{2} \sigma^{2}}}\right) .
$$

The proof of the lemma are omitted here, which can be derived straightly from some algebra operations, and readers interested in the proof details can refer to the literature [27]. According to Lemma 1, we can calculate the equation (4) and the equation (5) explicitly. The unconditional CDF of the life T can be directly obtained, and the unconditional PDF of the life $\mathrm{T}$ can be easily obtained by taking the derivative of the CDF with respect to $t$. The main results are summarized as follows.

Theorem 1. For the IG process model above, take into account the random effect of $\beta$ with $1 / \beta \sim N\left(\mu, \sigma^{2}\right)$, the following results hold:

$$
F_{T}(t)=\Phi\left(\sqrt{\frac{\eta}{\omega}} \cdot \frac{\sigma_{\beta} t-\mu_{\beta} \sigma_{\beta} \omega}{\sqrt{\sigma_{\beta}^{2}+\eta \omega}}\right)-\exp \left(2 \mu_{\beta} \eta t+\frac{2 \eta^{2} t^{2}}{\sigma_{\beta}^{2}}\right) \times \Phi\left(-\sqrt{\frac{\eta}{\omega}} \cdot \frac{\left(\sigma_{\beta}^{2}+2 \eta \omega\right) t+\mu_{\beta} \sigma_{\beta}^{2} \omega}{\sqrt{\sigma_{\beta}^{4}+\eta \omega \sigma_{\beta}^{2}}}\right)
$$

and

$$
\begin{aligned}
& f_{T}(t)= \sqrt{\frac{\eta}{\omega}} \frac{2 \sigma_{\beta}}{\sqrt{\sigma_{\beta}^{2}+\eta \omega}} \phi\left(\sqrt{\frac{\eta}{\omega}} \cdot \frac{\sigma_{\beta} t-\mu_{\beta} \sigma_{\beta} \omega}{\sqrt{\sigma_{\beta}^{2}+\eta \omega}}\right)-\exp \left(2 \mu_{\beta} \eta t+\frac{2 \eta^{2} t^{2}}{\sigma_{\beta}^{2}}\right) \\
& \times\left(2 \eta \mu_{\beta}+\frac{4 \eta^{2} t}{\sigma_{\beta}^{2}}\right) \Phi\left(-\sqrt{\frac{\eta}{\omega}} \cdot \frac{\left(\sigma_{\beta}^{2}+2 \eta \omega\right) t+\mu_{\beta} \sigma_{\beta}^{2} \omega}{\sqrt{\sigma_{\beta}^{4}+\eta \omega \sigma_{\beta}^{2}}}\right) . \\
& 4
\end{aligned}
$$


The focus of the above-mentioned formulae is on considering the life distribution on a population of the system. However, the aim of this study is to estimate the RUL distribution of an individual system using the degradation data through condition monitoring. In the following, our main objective is to derive the RUL distribution using the available degradation data in consideration of the random parameter $\beta$.

Let $\mathbf{Y}_{0: k}=\left\{y_{0}, y_{1}, \cdots, y_{k}\right\}$ denote the degradation observation at $0=t_{0}<t_{1}<\cdots<t_{k}$, which can be irregularly spaced, and $y_{i}=Y\left(t_{i}\right)$ represents the degradation measurement of a system at time $t_{i}$. Therefore, using the FHT of the degradation process, we define the RUL $R_{k}$ of a system at $t_{k}$ as

$$
R_{k}=\inf \left\{r_{k}>0: Y\left(t_{k}+r_{k}\right) \geq \omega \mid \mathbf{Y}_{0: k}\right\}
$$

On the basis of the definition of the RUL, conditional on the degradation data $\mathbf{Y}_{0: k}$ and $\beta$, the distribution function of the RUL can be obtained by the following theorem.

Theorem 2. For the IG process model above, and the definition of the RUL, given $\beta$ and the degradation data $\mathbf{Y}_{0: k}$, the following formulae hold:

$$
F_{R_{k} \beta \beta}\left(r_{k} \mid \beta, \mathbf{Y}_{0: k}\right)=\Phi\left[\sqrt{\frac{\eta}{\omega-y_{k}}}\left(r_{k}-\frac{\omega-y_{k}}{\beta}\right)\right]-\exp \left(\frac{2 \eta r_{k}}{\beta}\right) \Phi\left[-\sqrt{\frac{\eta}{\omega-y_{k}}}\left(r_{k}+\frac{\omega-y_{k}}{\beta}\right)\right],
$$

and

$$
f_{R_{k} \beta}\left(r_{k} \mid \beta, \mathbf{Y}_{0: k}\right)=2 \sqrt{\frac{\eta}{\omega-y_{k}}} \phi\left[\sqrt{\frac{\eta}{\omega-y_{k}}}\left(r_{k}-\frac{\omega-y_{k}}{\beta}\right)\right]-\frac{2 \eta}{\beta} \exp \left[\frac{2 \eta r_{k}}{\beta}\right] \Phi\left[-\sqrt{\frac{\eta}{\omega-y_{k}}}\left(r_{k}+\frac{\omega-y_{k}}{\beta}\right)\right] .
$$

When the parameter $\beta$ is fixed, according to the definition of the RUL and the equation (3), the mean of the RUL can be easily obtained as follows:

$$
E\left(R_{k} \mid \beta\right)=\left(\frac{\omega-y_{k}}{\beta}+\frac{\beta}{\eta}\right) \Phi\left(\frac{\sqrt{\eta\left(\omega-y_{k}\right)}}{\beta}\right)+\sqrt{\frac{\omega-y_{k}}{\eta}} \phi\left(\frac{\sqrt{\eta\left(\omega-y_{k}\right)}}{\beta}\right)-\frac{\beta}{2 \eta} .
$$

In the case of taking the random effect of $\beta$ into consideration, on the basis of Theorem 1 and Theorem 2 , we have the following results for the RUL estimation with random effect.

Theorem 3. For the IG process model above, and the definition of the RUL, given the degradation data $\mathbf{Y}_{0: k}$ and $1 / \beta \sim N\left(\mu_{\beta}, 1 / \sigma_{\beta}^{2}\right)$, the following formulae hold:

$$
\begin{aligned}
F_{R_{k}}\left(r_{k} \mid \mathbf{Y}_{0: k}\right)= & \Phi\left(\sqrt{\frac{\eta}{\omega-y_{k}}} \cdot \frac{\sigma_{\beta} r_{k}-\mu_{\beta} \sigma_{\beta}\left(\omega-y_{k}\right)}{\sqrt{\sigma_{\beta}^{2}+\eta\left(\omega-y_{k}\right)}}\right)-\exp \left(2 \mu_{\beta} \eta r_{k}+\frac{2 \eta^{2} r_{k}^{2}}{\sigma_{\beta}^{2}}\right) \\
& \times \Phi\left(-\sqrt{\frac{\eta}{\omega-y_{k}}} \cdot \frac{\left(\sigma_{\beta}^{2}+2 \eta\left(\omega-y_{i}\right)\right) r_{k}+\mu_{\beta} \sigma_{\beta}^{2}\left(\omega-y_{k}\right)}{\sqrt{\sigma_{\beta}^{4}+\eta\left(\omega-y_{k}\right) \sigma_{\beta}^{2}}}\right),
\end{aligned}
$$

and

$$
\begin{aligned}
f_{R_{k}}\left(r_{k} \mid \mathbf{Y}_{0: k}\right)= & \sqrt{\frac{\eta}{\omega-y_{k}}} \frac{2 \sigma_{\beta}}{\sqrt{\sigma_{\beta}^{2}+\eta\left(\omega-y_{k}\right)}} \phi\left(\sqrt{\frac{\eta}{\omega-y_{k}}} \cdot \frac{\sigma_{\beta} r_{k}-\mu_{\beta} \sigma_{\beta}\left(\omega-y_{k}\right)}{\sqrt{\sigma_{\beta}^{2}+\eta\left(\omega-y_{k}\right)}}\right)-\left(2 \eta \mu_{\beta}+\frac{4 \eta^{2} r_{k}}{\sigma_{\beta}^{2}}\right) \\
& \times \exp \left(2 \mu_{\beta} \eta r_{k}+\frac{2 \eta^{2} r_{k}^{2}}{\sigma_{\beta}^{2}}\right) \Phi\left(-\sqrt{\frac{\eta}{\omega-y_{k}}} \cdot \frac{\left(\sigma_{\beta}^{2}+2 \eta\left(\omega-y_{k}\right)\right) r_{k}+\mu_{\beta} \sigma_{\beta}^{2}\left(\omega-y_{k}\right)}{\sqrt{\sigma_{\beta}^{4}+\eta\left(\omega-y_{k}\right) \sigma_{\beta}^{2}}}\right) .
\end{aligned}
$$


Additionally, when the random effect of $\beta$ is considered, the mean of the RUL can be derived as follows:

$$
\begin{aligned}
E\left(R_{k}\right) & =E_{\beta}\left[E\left(R_{k} \mid \beta\right)\right] \\
& =E_{\beta}\left[\left(\frac{\omega-y_{k}}{\beta}+\frac{\beta}{\eta}\right) \Phi\left(\frac{\sqrt{\eta\left(\omega-y_{k}\right)}}{\beta}\right)+\sqrt{\frac{\omega-y_{k}}{\eta}} \phi\left(\frac{\sqrt{\eta\left(\omega-y_{k}\right)}}{\beta}\right)-\frac{\beta}{2 \eta}\right] \\
& =\int_{0}^{\omega-y_{k}} \sqrt{(\eta y)^{-1}+\sigma_{\beta}^{2}} \phi\left(\sqrt{(\eta y)^{-1}+\sigma_{\beta}^{2}} \mu_{\beta}\right)+\mu_{\beta} \Phi\left(\sqrt{(\eta y)^{-1}+\sigma_{\beta}^{2}} \mu_{\beta}\right) d y .
\end{aligned}
$$

Although the analytical expression of the integral above is not available, we can easily see that the traditional numerical integration method is able to approximate the mean of the RUL properly, such as composite Simpson rule, Romberg integration, Gauss-Legendre integration and so on.

\section{Statistical Inference}

In this section, we proceed to the issue of estimating the model parameter to implement the RUL estimation of the system based on the available degradation data. Assume a tested system is monitored at ordered times $t_{0}, t_{1}, \cdots, t_{n}$, where $n$ denotes the available number of the degradation data. Let $\Delta y_{i}=y_{i}-y_{i-1}$ denote the degradation increment from time $t_{i-1}$ to $t_{i}$ for a tested system. According to the nature of IG process, under the condition of a fixed parameter $\beta$, the sampling distribution of $\mathbf{Y}_{0: k}$ can be written by the following expression:

$$
p\left(\mathbf{Y}_{0: k} \mid \beta\right)=\prod_{i=1}^{k}\left(\frac{\eta \Delta t_{i}{ }^{2}}{2 \pi \Delta y_{i}{ }^{3}}\right)^{1 / 2} \exp \left[-\frac{\eta\left(\Delta y_{i}-\beta \Delta t_{i}\right)^{2}}{2 \beta^{2} \Delta y_{i}}\right]
$$

where $\Delta t_{i}=t_{i}-t_{i-1}$ represents the time gap. In order to emphasize the constant updating of model parameters along with the available degradation data $\mathbf{Y}_{0: k}$, we redefine the notation of unknown parameters $\mu_{\beta}, \sigma_{\beta}^{2}, \eta$ as $\mu_{\beta, k}, \sigma_{\beta, k}^{2}, \eta_{k}$ respectively. As mentioned above, the prior distribution of $1 / \beta$ is assumed to follow a normal distribution with mean $\mu_{\beta, k}$ and variance $1 / \sigma_{\beta, k}^{2}$. It is worth noting that such prior distribution practically falls into the conjugate family of $p\left(\mathbf{Y}_{0: k} \mid \beta\right)$. As a result, the posterior estimate of $1 / \beta$ conditional on $p\left(\mathbf{Y}_{0: k} \mid \beta\right)$ is still normal distribution with mean $\mu_{0, k}$ and variance $1 / \sigma_{0, k}^{2}$. According to the Bayesian rule, the posterior distribution of $1 / \beta$ can be updated by the following expression:

$$
\begin{aligned}
p\left(\frac{1}{\beta} \mid \mathbf{Y}_{0: k}\right) & \propto \exp \left[-\frac{\sigma_{\beta, k}^{2}\left(1-\beta \mu_{\beta, k}\right)}{2 \beta^{2}}\right] \prod_{i=1}^{k} \exp \left[-\frac{\eta_{k}\left(\Delta y_{i}-\beta \Delta t_{i}\right)^{2}}{2 \beta^{2} \Delta y_{i}}\right] \\
& \propto \exp \left[-\frac{\eta_{k} y_{k}+\sigma_{\beta, k}^{2}}{2}\left(\frac{1}{\beta}-\frac{\eta_{k} t_{k}+\mu_{\beta, k} \sigma_{\beta, k}^{2}}{\eta_{k} y_{k}+\sigma_{\beta, k}^{2}}\right)\right] \\
& \sim N\left(\mu_{0, k}, \frac{1}{\sigma_{0, k}^{2}}\right)
\end{aligned}
$$

with

$$
\begin{aligned}
\mu_{0, k} & =\frac{\eta_{k} t_{k}+\mu_{\beta, k} \sigma_{\beta, k}^{2}}{\eta_{k} y_{k}+\sigma_{\beta, k}^{2}}, \\
\sigma_{0, k}^{2} & =\eta_{k} y_{k}+\sigma_{\beta, k}^{2} .
\end{aligned}
$$

It is noted that the posterior estimate of $1 / \beta$ can be updated once the new degradation observation is available. For this reason, the RUL can be adaptively estimated in the light of Theorem 3, which makes it closer to the actual RUL of the system with less uncertainty. When $1 / \beta$ is fixed, the log-likelihood function with respect to $\mathbf{Y}_{0: k}$ can be expressed as follow:

$$
\mathcal{L}\left(\Theta \mid \mathbf{Y}_{0: k}, \frac{1}{\beta}\right)=\frac{k}{2}\left(\ln \eta_{k}-\ln 2 \pi\right)+\sum_{i=1}^{k} \ln \Delta t_{i}-\frac{3}{2} \sum_{i=1}^{k} \ln \Delta y_{i}-\sum_{i=1}^{k} \frac{\eta_{k}\left(\Delta y_{i}-\beta \Delta t_{i}\right)^{2}}{2 \beta^{2} \Delta y_{i}}
$$


It is difficult to obtain the MLE of the model parameters above, because we must maximize the log-likelihood function (20). However, it is very tough for direct constrained optimization of the log-likelihood function owing to the unobservability of $1 / \beta$, which usually can not converge to a solution. The EM algorithm [26] provides a possible way for resolving this difficulty, which is a general method of determining the MLE of the parameters of a potential distribution when the likelihood function contains unobserved latent variables $[2,14]$. The fundamental idea of the EM algorithm is to replace the latent variable $1 / \beta$ with its conditional expectation, where the parameter updates in each step can be acquired in a closed form, or a simple manner. Let $\Theta_{k}=\left(\mu_{\beta, k}, \sigma_{\beta, k}^{2}, \eta_{k}\right)$ be the unknown parameter vector, and denote $\hat{\Theta}_{k}^{(j)}=\left(\mu_{\beta, k}^{(j)}, \sigma_{\beta, k}^{2(j)}, \eta_{k}^{(j)}\right)$ as the parameter estimates in the $j$ th step for the EM algorithm based on the available degradation data $\mathbf{Y}_{0: k}$. When both $Y$ and $1 / \beta$ are regarded as be observable, the complete log-likelihood function can be expressed as follow:

$$
\begin{aligned}
\mathcal{L}_{k}\left(\Theta_{k} \mid \mathbf{Y}_{0: k}\right)=\ln p\left(\mathbf{Y}_{0: k}, \frac{1}{\beta} \mid \Theta_{k}\right)= & \ln p\left(\mathbf{Y}_{0: k} \mid \frac{1}{\beta}, \Theta_{k}\right)+\ln p\left(\frac{1}{\beta} \mid \Theta_{k}\right) \\
= & \frac{k}{2} \ln \eta_{k}-\frac{k+1}{2} \ln 2 \pi+\sum_{i=1}^{k} \ln \Delta t_{i}-\frac{3}{2} \sum_{i=1}^{k} \ln \Delta y_{i} \\
& -\sum_{i=1}^{k} \frac{\eta_{k}\left(\Delta y_{i}-\beta \Delta t_{i}\right)^{2}}{2 \beta^{2} \Delta y_{i}}+\frac{1}{2} \ln \sigma_{\beta, k}^{2}-\frac{\sigma_{\beta, k}^{2}}{2}\left(\frac{1}{\beta}-\mu_{\beta, k}\right)^{2} .
\end{aligned}
$$

In the following, we use the EM algorithm to find the MLE of the unknown parameters iteratively, that is, the estimate of $\Theta_{k}$ can be achieved by the E-step and M-step. The EM algorithm firstly finds the expected value of the complete log-likelihood function with respect to the latent variable $1 / \beta$, and then the M-step is to maximize the expectation computed in the E-step. To be specific, the E-step computes the expectation $Q\left(\Theta_{k} \mid \mathbf{Y}_{0: k}, \hat{\Theta}_{k}^{(j)}\right)$ of $\mathcal{L}_{k}\left(\Theta_{k} \mid \mathbf{Y}_{0: k}\right)$ with respect to $1 / \beta$, we can obtain

$$
\begin{aligned}
Q\left(\Theta_{k} \mid \mathbf{Y}_{0: k}, \hat{\Theta}_{k}^{(j)}\right)= & E_{\frac{1}{\beta} \mid \mathbf{Y}_{0: k}, \hat{\Theta}_{k}^{(j)}}\left\{\mathcal{L}_{k}\left(\Theta_{k} \mid \mathbf{Y}_{0: k}\right)\right\} \\
= & \frac{k}{2} \ln \eta_{k}-\frac{k+1}{2} \ln 2 \pi+\sum_{i=1}^{k} \ln \Delta t_{i}-\frac{3}{2} \sum_{i=1}^{k} \ln \Delta y_{i}+\frac{1}{2} \ln \sigma_{\beta, k}^{2} \\
& -\frac{\eta_{k}}{2} \sum_{i=1}^{k}\left[\left(\left(\mu_{0, k}^{(j)}\right)^{2}+\frac{1}{\sigma_{0, k}^{2(j)}}\right) \Delta y_{i}-2 \mu_{0, k}^{(j)} \Delta t_{i}+\frac{\Delta t_{i}^{2}}{\Delta y_{i}}\right]-\frac{\sigma_{\beta, k}^{2}}{2}\left[\left(\mu_{0, k}^{(j)}\right)^{2}+\frac{1}{\sigma_{0, k}^{2(j)}}-2 \mu_{0, k}^{(j)} \mu_{\beta, k}+\mu_{\beta, k}^{2}\right] .
\end{aligned}
$$

In the M-step, set $\frac{\partial Q\left(\Theta_{k} \mid \mathbf{Y}_{0: k}, \hat{\Theta}_{k}^{(j)}\right)}{\partial \Theta_{k}}=0$, we can obtain the current parameter estimates $\hat{\Theta}_{k}^{(j+1)}$ as follows:

$$
\begin{aligned}
\mu_{\beta, k}^{(j+1)} & =\mu_{0, k}^{(j)}, \\
\sigma_{\beta, k}^{2(j+1)} & =\sigma_{0, k}^{2(j)}, \\
\eta_{k}^{(j+1)} & =\left\{\frac{1}{k} \sum_{i=1}^{k}\left[\left(\left(\mu_{0, k}^{(j)}\right)^{2}+\frac{1}{\sigma_{0, k}^{2(j)}}\right) \Delta y_{i}-2 \mu_{0, k}^{(j)} \Delta t_{i}+\frac{\Delta t_{i}^{2}}{\Delta y_{i}}\right]\right\}^{-1} .
\end{aligned}
$$

It's worth noting that the M-step can be resolved analytically and obtain the unique maximum point. In the following, we will give the detailed derivation procedure by using the idea of $[22,23]$. Firstly, taking $\partial^{2} Q\left(\Theta_{k} \mid \mathbf{Y}_{0: k}, \hat{\Theta}_{k}^{(j)}\right) / \partial \Theta_{k} \partial \Theta_{k}^{T}$, we can obtain the following formula:

$$
\frac{\partial^{2} Q\left(\Theta_{k} \mid \mathbf{Y}_{0: k}, \hat{\Theta}_{k}^{(j)}\right)}{\partial \Theta_{k} \partial \Theta_{k}^{T}}=\left(\begin{array}{ccc}
-\sigma_{\beta, k}^{2} & \mu_{0, k}^{(j)}-\mu_{\beta, k} & 0 \\
\mu_{0, k}^{(j)}-\mu_{\beta, k} & -\frac{1}{2 \sigma_{\beta, k}^{4}} & 0 \\
0 & 0 & -\frac{k}{2 \eta_{k}^{2}}
\end{array}\right)
$$

In fact, we can demonstrate that the matrix in (26) is negative definite at $\Theta_{k}=\hat{\Theta}_{k}^{(j+1)}$, by computing the order principal minor determinant at $\Theta_{k}=\hat{\Theta}_{k}^{(j+1)}$ as follows:

$$
\left.\mathbf{H}_{1}\right|_{\Theta_{k}=\hat{\Theta}_{k}^{(j+1)}}=-\sigma_{\beta, k}^{2(j+1)}<0
$$




$$
\begin{aligned}
\left.\mathbf{H}_{2}\right|_{\Theta_{k}=\Theta_{k}^{(j+1)}} & =\frac{1}{2 \sigma_{\beta, k}^{2}}-\left.\left(\mu_{0, k}^{(j)}-\mu_{\beta, k}\right)^{2}\right|_{\Theta_{k}=\hat{\Theta}_{k}^{(j+1)}}=\frac{1}{2 \sigma_{\beta, k}^{2(j+1)}}>0 \\
\left.\mathbf{H}_{3}\right|_{\Theta_{k}} & =-\left.\frac{k}{2 \eta_{k}^{2}} \mathbf{H}_{2}\right|_{\Theta_{k}=\Theta_{k}^{(j+1)}}=-\frac{k}{2\left(\eta_{k}^{(j+1)}\right)^{2}} \mathbf{H}_{2}<0
\end{aligned}
$$

This completes the proof that the matrix in (26) is negative definite at $\Theta_{k}=\hat{\Theta}_{k}^{(j+1)}$. According to the sufficient condition of the existent extreme value of multivariate function, $\hat{\Theta}_{k}^{(j+1)}$ is located at a maximum point, and $\Theta_{k}=\hat{\Theta}_{k}^{(j+1)}$ is unique because it is the only solution satisfying $\frac{\partial Q\left(\Theta_{k} \mid \mathbf{Y}_{0: k}, \hat{\Theta}_{k}^{(j)}\right)}{\partial \Theta_{k}}=0$. It can be found that the parameter updates in each iteration of the EM algorithm have explicit formulae, i.e., each iteration of the EM algorithm can be performed with a single computation. In short, the above steps are iterated by multiple times to generate a sequence $\left\{\hat{\Theta}_{k}^{(0)}, \hat{\Theta}_{k}^{(1)}, \hat{\Theta}_{k}^{(2)}, \cdots\right\}$ of increasingly good approximations $\hat{\Theta}_{k}=\left(\hat{\mu}_{\beta, k}, \hat{\sigma}_{\beta, k}^{2}, \hat{\eta}_{k}\right)$ to $\Theta_{k}$. The iterations are generally terminated using a standard criterion such as the difference between $\hat{\Theta}_{k}^{(j+1)}$ and $\hat{\Theta}_{k}^{(j)}$ falling below a predefined threshold.

\section{Illustrative examples}

In this section, we will provide a simulated example as well as a case study to validate the effectiveness and superiority of the proposed RUL estimation approach and parameter estimation procedure. We firstly provide a simulated example to demonstrate the proposed statistical inference procedure.

\subsection{Simulated example}

A simulation study is conducted to illustrate the proposed parameter estimation approach to be effective and feasible. In the simulation, the degradation data are generated based on the following parameter values: $\mu_{\beta}=0.5$, $1 / \sigma_{\beta}^{2}=10^{-6}$ and $\eta=5$. The inspection times are taken from 0 to 40 with the measured frequency of $\Delta t=1 / 2$, and the failure threshold is chosen to be $\omega=80$ with the corresponding true life $T=40$. The simulated degradation data are shown in Figure 1.

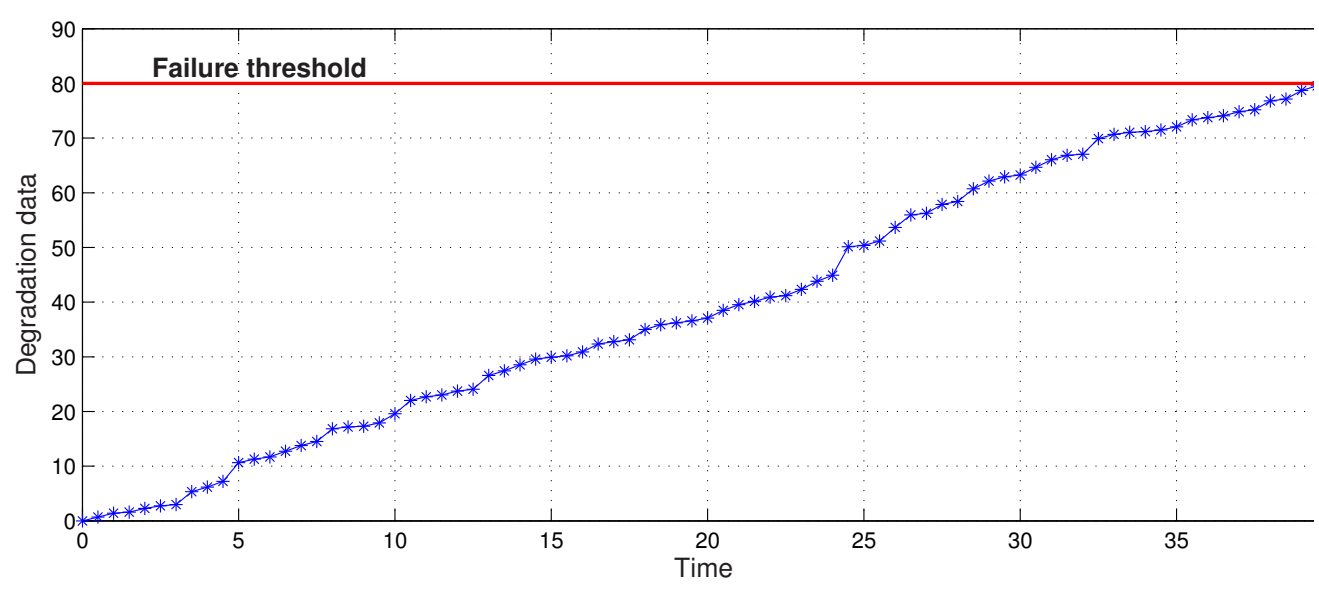

Figure 1. The simulated degradation data.

As mentioned in the above section, the EM algorithm is used to find the MLE $\hat{\Theta}_{k}=\left(\hat{\mu}_{\beta, k}, \hat{\sigma}_{\beta, k}^{2}, \hat{\eta}_{k}\right)$ of the unknown parameter vector $\Theta_{k}$ iteratively using the simulated degradation data. Figure 2 provides the real-time estimated results of the above three parameters at different inspection times. It can be easily seen that the estimated results for each parameter continually approximate the true parameter value as the available degradation data increase. The main reason for this is that the estimated results for each parameter can be real-time updated based on the newly obtained degradation data as a result of the Baysian updating mechanism in parameter estimation process. In addition, we can find that the EM algorithm has good performance on convergence speed in that the parameter updates of each iteration of the EM algorithm have explicit expression, i.e., each iteration can be conducted with one single calculation, which 

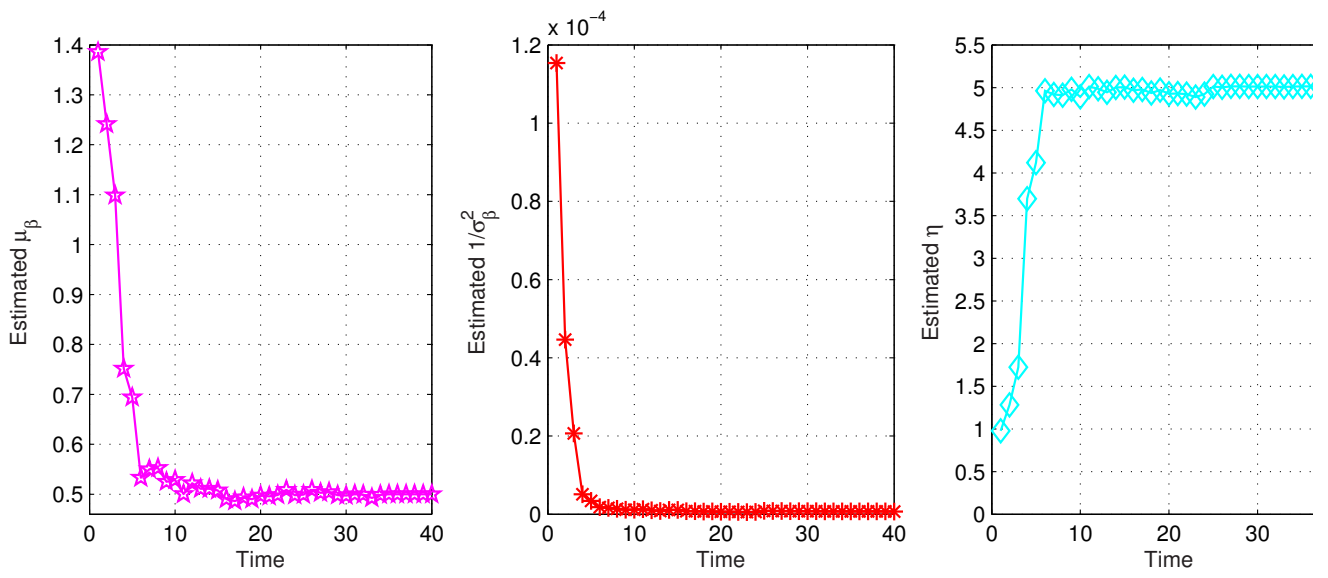

Figure 2. The updated parameters at different inspection times.

results in a particularly fast and simple estimation procedure. The result reveals that the updated parameters for our model can achieve a fast convergence as the observed degradation data increase.

Table 1. Percentage increase in laser operating current over time.

\begin{tabular}{|c|c|c|c|c|c|c|c|c|c|c|c|c|c|c|c|}
\hline No. & 1 & 2 & 3 & 4 & 5 & 6 & 7 & 8 & 9 & 10 & 11 & 12 & 13 & 14 & 15 \\
\hline 0 & 0 & 0 & 0 & 0 & 0 & 0 & 0 & 0 & 0 & 0 & 0 & 0 & 0 & 0 & 0 \\
\hline 250 & 0.47 & 0.71 & 0.71 & 0.36 & 0.27 & 0.36 & 0.36 & 0.46 & 0.51 & 0.41 & 0.44 & 0.39 & 0.30 & 0.44 & 0.50 \\
\hline 500 & 0.93 & 1.22 & 1.17 & 0.62 & 0.61 & 1.39 & 0.92 & 1.07 & 0.93 & 1.49 & 1.00 & 0.80 & 0.74 & 0.70 & 0.83 \\
\hline 750 & 2.11 & 1.90 & 1.73 & 1.36 & 1.11 & 1.95 & 1.21 & 1.42 & 1.57 & 2.38 & 1.57 & 1.35 & 1.52 & 1.05 & 1.29 \\
\hline 1000 & 2.72 & 2.30 & 1.99 & 1.95 & 1.77 & 2.86 & 1.46 & 1.77 & 1.96 & 3.00 & 1.96 & 1.74 & 1.85 & 1.35 & 1.52 \\
\hline 1250 & 3.51 & 2.87 & 2.53 & 2.30 & 2.06 & 3.46 & 1.93 & 2.11 & 2.59 & 3.84 & 2.51 & 2.98 & 2.39 & 1.80 & 1.91 \\
\hline 1500 & 4.34 & 3.75 & 2.97 & 2.95 & 2.58 & 3.81 & 2.39 & 2.40 & 3.29 & 4.50 & 2.84 & 3.59 & 2.95 & 2.55 & 2.27 \\
\hline 1750 & 4.91 & 4.42 & 3.30 & 3.39 & 2.99 & 4.53 & 2.68 & 2.78 & 3.61 & 5.25 & 3.47 & 4.03 & 3.51 & 2.83 & 2.78 \\
\hline 2000 & 5.48 & 4.99 & 3.94 & 3.79 & 3.38 & 5.35 & 2.94 & 3.02 & 4.11 & 6.26 & 4.01 & 4.44 & 3.92 & 3.39 & 3.42 \\
\hline 2250 & 5.99 & 5.51 & 4.16 & 4.11 & 4.05 & 5.92 & 3.42 & 3.29 & 4.60 & 7.05 & 4.51 & 4.79 & 5.03 & 3.72 & 3.78 \\
\hline 2500 & 6.72 & 6.07 & 4.45 & 4.50 & 4.63 & 6.71 & 4.09 & 3.75 & 4.91 & 7.80 & 4.80 & 5.22 & 5.47 & 4.09 & 4.11 \\
\hline 2750 & 7.13 & 6.64 & 4.89 & 4.72 & 5.24 & 7.70 & 4.58 & 4.16 & 5.34 & 8.32 & 5.20 & 5.48 & 5.84 & 4.83 & 4.38 \\
\hline 3000 & 8.00 & 7.16 & 5.27 & 4.98 & 5.62 & 8.61 & 4.84 & 4.76 & 5.84 & 8.93 & 5.66 & 5.96 & 6.50 & 5.41 & 4.63 \\
\hline 3250 & 8.92 & 7.78 & 5.69 & 5.28 & 6.04 & 9.15 & 5.11 & 5.16 & 6.40 & 9.55 & 6.20 & 6.23 & 6.94 & 5.76 & 5.38 \\
\hline 3500 & 9.49 & 8.42 & 6.02 & 5.61 & 6.32 & 9.95 & 5.57 & 5.46 & 6.84 & 10.45 & 6.54 & 6.99 & 7.39 & 6.14 & 5.84 \\
\hline 3750 & 9.87 & 8.91 & 6.45 & 5.95 & 7.10 & 10.49 & 6.11 & 5.81 & 7.20 & 11.28 & 6.96 & 7.37 & 7.85 & 6.51 & 6.16 \\
\hline 4000 & 10.94 & 9.28 & 6.88 & 6.14 & 7.59 & 11.01 & 7.17 & 6.24 & 7.88 & 12.21 & 7.22 & 7.88 & 8.09 & 6.88 & 6.62 \\
\hline
\end{tabular}

\subsection{Case study}

In this section, we consider the degradation data of the laser device, which are taken from Meeker and Escobar [19]. This dataset has been extensively studied in degradation modeling [15, 16, 30, 31]. The existing literatures have been demonstrated that the Wiener and Gamma process do not fit this dataset well.

The degradation of some laser devices leads to the deterioration of light output during their whole life cycle. To remedy the influences of the degradation of a laser on its light output, the laser generally increases the operating current by using a feedback mechanism to maintain nearly constant light output. In the degradation test described by [19], 15 units are tested for the operating current over time with measure frequency 250 hours. A laser is announced to have failed if the percentage of its operating current exceeds a predefined threshold level $\omega=10 \%$, so it is considered as the degradation data of the laser. Table 1 shows the degradation data of 15 tested units along with the terminal time 4000 hours. Note that the observation time points are recorded at every 250 hours, this leads to the inability to 
know the true life, i.e. the time arriving the failure threshold. We can observe from Table 1 that the percentage value of the sixth sample is 9.95 at the 3500th hour, which is quite close to the predefined failure threshold $\omega=10 \%$. As a result, for the sake of contrastive analysis below, we assume the failure of the sixth test sample occurs when there is $\omega=9.95 \%$ increase in the operating current, as shown in Figure 3. From the degradation data shown in Figure 3 , it is reasonable to use a linear function in the IG process to model the degradation process of the laser device. In the following, we will use the degradation data to demonstrate the effectiveness of the proposed RUL estimation approach.

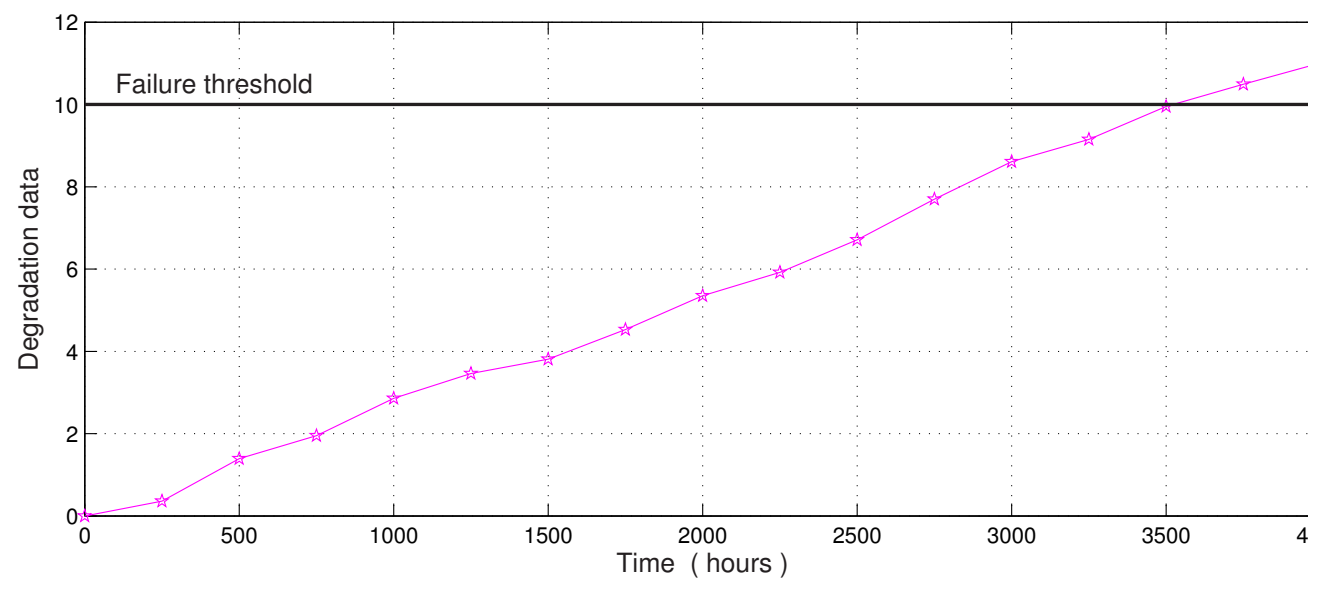

Figure 3. The degradation data of the sixth test sample.

To assess the accuracy of the RUL estimation, the $\alpha-\lambda$ performance metric [20] is adopted to quantify the estimated results of mean RUL, which is defined by

$$
(1-\alpha)\left(t_{E O L}-t\right) \leq r_{p}(t) \leq(1+\alpha)\left(t_{E O L}-t\right)
$$

where $t=t_{j}+\lambda\left(t_{E O L}-t_{j}\right), t_{j}$ is the first estimation time point, $\lambda$ represents the window modifier, $\alpha$ represents the accuracy modifier and $r_{p}(t)$ is the estimated RUL at time $t$. The performance metric is mainly used to measure whether the the RUL estimation approach performs within desired error tolerance (specified by $\alpha$ ) of the true mean RUL at any specified time point or not. Additionally, it is worth noting that the alpha bounds are not the uncertainty bounds of the estimated RUL, which only represents its uncertainty.

According to the proposed RUL estimation approach, we calculate the $\alpha-\lambda$ performance metric at several time points, and RUL estimation results without the incorporation of random effect are also provided to show the necessary of random effect and Bayesian parameter updating. The comparative results are summarized in Figure 4, in which the blue asterisks and the red pentacles represent estimated mean RULs with and without random effect at distinct time points respectively. It is easily seen that using the RUL estimation approach with random effect can obtain $80 \%$ accuracy $(\alpha=0.2)$ right from the 500th hour onwards $(\lambda=0)$ and the acceptable limit is wider for early estimation than for those that are late in terms of error bounds. This indicates that the precision of the estimated RUL is improved as the available degradation data increase. However, if the random effect is not considered in the process of degradation modeling and RUL estimation, the estimated RULs are nearly beyond acceptable limits, especially in the early predictions. It is primarily because the RUL estimation approach with random effect can real-time updating the RUL distribution to make the RUL estimation more accurate as the new degradation data are available. Therefore, RUL estimation approach with random effect significantly performs better than RUL estimation approach without random effect, it demonstrates the necessary of random effect and Bayesian parameter updating. In addition, Figure 5 also provides the several estimated PDFs of the RUL with and without the incorporation of random effect, in which the red diamonds represent the true RULs, and the blue lines and the magenta lines represent the estimated PDFs with and without random effect respectively. It can be found that the range of estimated PDFs with random effect can cover the true RULs, and the estimated PDFs become narrower as the more degradation data are collected, in contrast with 


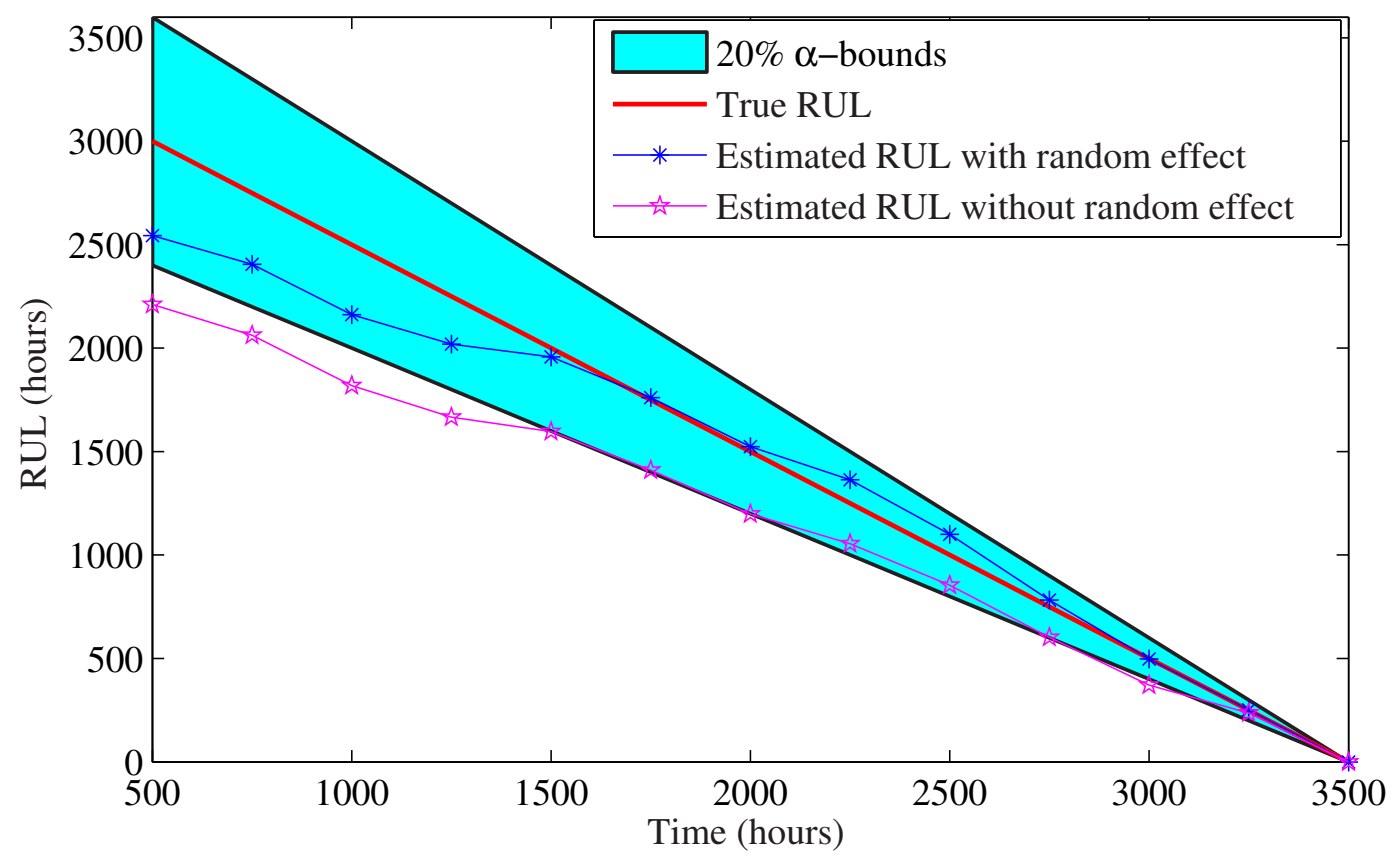

Figure 4. $\alpha-\lambda$ performance for the sixth sample with and without random effects. The shaded cyan area is the $80 \%$ accuracy cone $(\alpha=0.2)$.

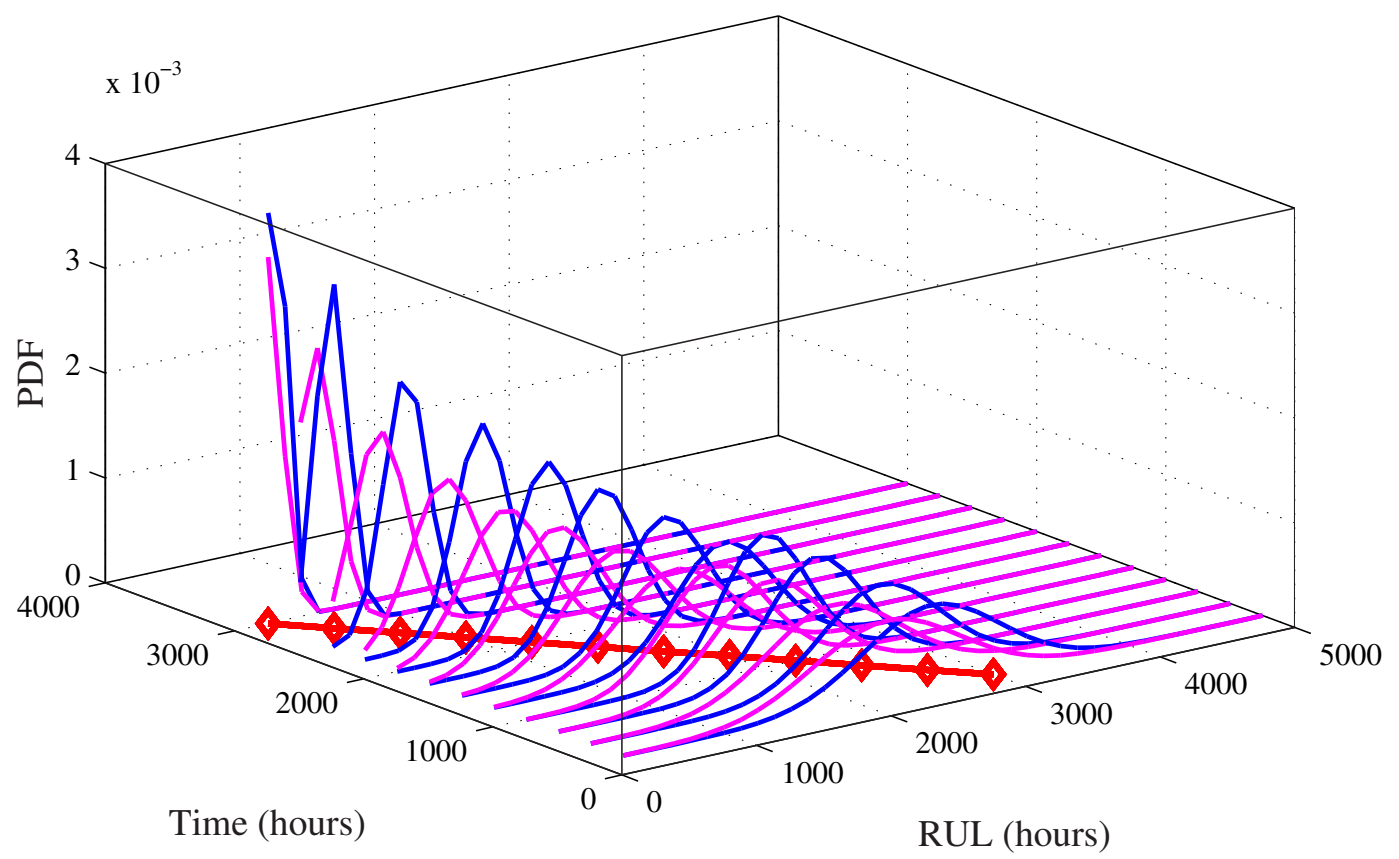

Figure 5. The estimated PDFs of the RUL with and without random effects. 
estimated PDFs without the incorporation of random effect. This further demonstrates that our proposed approach can estimate the RUL with lesser uncertainty and higher accuracy.

\section{Conclusions}

This paper presents an adaptive RUL estimation approach based on an IG process with the random effect. In our proposed approach, the random effect is used to characterize the unit-to-unit heterogeneities, and the uncertainty associated with the random effect is incorporated into the real-time estimated RUL distribution. In the parameter estimation, EM algorithm is employed to estimate the model parameters. With the aid of the Bayesian updating mechanism, the random parameter can be real-time updated by the Bayesian rule using the available degradation observation. For this reason, the estimated PDFs of the RULs can be dynamically updated on the basis of the arrivals of new degradation data from the interested system. In addition, the proposed approach can obtain an explicit expression of the RUL distribution, and there also exists the moment of the obtained RUL distribution. The illustrative examples demonstrated that the adaptive RUL estimation approach can increase the RUL estimation accuracy, and the uncertainty of the estimated RUL is improved as the more degradation data become available.

\section{Acknowledgments}

The authors would like to express their sincere gratitude to the anonymous referees for many friendly and helpful suggestions, which led to great deal of improvement of the original manuscript.

The work of D. Pan was partly supported by the National Natural Science Foundation of China under Grants 11501005 and 11301002, the Academic and Technology Leaders to Introduction Projects of Anhui University. The work of J. B. Liu was partly supported by the Natural Science Foundation for the Higher Education Institutions of Anhui Province of China under Grant no. KJ2015A331 and the National Science Foundation of China under Grants 11471016 and 11401004 . The work of J. Cao was partly supported by the National Natural Science Foundation of China under Grant 61272530, the Natural Science Foundation of Jiangsu Province of China under Grant BK2012741, the Specialized Research Fund for the Doctoral Program of Higher Education under Grants 20110092110017 and 20130092110017.

\section{References}

[1] M. J. Daigle, K. Goebel, Model-based prognostics with concurrent damage progression processes, IEEE Trans. Syst. Man Cybern. Syst. 43(3) (2013) 535-546.

[2] A. P. Dempster, N. M. Laird, D. B. Rubin, Maximum likelihood from incomplete data via the EM algorithm, J. Roy. Statist. Soc. Ser. B 39(1) (1977) 1-38.

[3] N. Gebraeel, A. Elwany, J. Pan, Residual life predictions in the absence of prior degradation knowledge, IEEE Trans. Rel. 58(1) (2009) 106-111.

[4] N. Gebraeel, M. A. Lawley, R. Li, K.J. Ryan, Residual-life distributions from component degradation signals: a Bayesian approach, IIE Trans. 37 (2005) 543-557.

[5] N. Gebraeel, J. Pan, Prognostic degradation models for computing and updating residual life distributions in a time-varying environment, IEEE Trans. Rel. 57(4) (2008) 539-550.

[6] K. Javed, R. Gouriveau, N. Zerhouni, P. Nectoux, Enabling health monitoring approach based on vibration data for accurate prognostics, IEEE Trans. Ind. Electron. 62(1) (2015) 647-656.

[7] K. Javed, R. Gouriveau, N. Zerhouni, A new multivariate approach for prognostics based on extreme learning machine and fuzzy clustering, IEEE Trans. Cybern. DOI: 10.1109/TCYB.2014.2378056, (2015).

[8] G. Jin, D. E. Matthews, Z. Zhou, A Bayesian framework for on-line degradation assessment and residual life prediction of secondary batteries in spacecraft, Reliab. Eng. Syst. Saf. 113 (2013) 7-20.

[9] P. K. Kankar, S. C. Sharma, S. P. Harsha, Rolling element bearing fault diagnosis using wavelet transform, Neurocomputing 74 (2011) 1638-1645.

[10] Z. W. Liu, H. R. Cao, X. F. Chen, et al., Multi-fault classification based on wavelet SVM with PSO algorithm to analyze vibration signals from rolling element bearings, Neurocomputing 157 (2015) 208-222.

[11] Z. Y. Liu, X. B. Ma, Y. Yang, Y. Zhao, Reliability modeling for systems with multiple degradation processes using Inverse Gaussian process and copulas, Mathematical Problems in Eng. 2014, Article ID 829597, 10 pages (2014).

[12] K. Le Son, M. Fouladirad, A. Barros, E. Levrat,B. Iung, Remaining useful life estimation based on stochastic deterioration models: a comparative study, Reliab. Eng. Syst. Saf. 112 (2013) 165-175.

[13] H. Li, D. H. Pan, C. L. P. Chen, Intelligent prognostics for battery health monitoring using the mean entropy and relevance vector machine, IEEE Trans. Syst. Man Cybern. Syst. 44(7) (2014) 7851-7862. 
[14] T. S. Ng, An application of the EM algorithm to degradation modeling, IEEE Trans. Rel. 57(1) (2008) 2-13.

[15] C. Peng, Inverse Gaussian processes with random effects and explanatory variables for degradation data, Technometrics 57(1) (2014) 100-111.

[16] W. W. Peng, Y. F. Li, Y. J. Yang, H. Z. Huang, M. J. Zuo, Inverse Gaussian process models for degradation analysis: A Bayesian perspective, Reliab. Eng. Syst. Saf. 130 (2014) 175-189.

[17] C. Y. Peng, S. T. Tseng, Mis-specification analysis of linear degradation models, IEEE Trans. Rel. 58(3) (2009) $444-455$.

[18] H. Qin, S. Zhang, W. Zhou, Inverse Gaussian process-based corrosion growth modeling and its application in the reliability analysis for energy pipelines, Front Struct. Civil. Eng. 7(3) (2013) 276-287.

[19] W. Q. Meeker, L. A. Escobar, Statistical methods for reliability data, New York: Wiley (1998).

[20] A. Saxena, J. Celaya, B. Saha, S. Saha, and K. Goebel, Metrics for offline evaluation of prognostics performance, Int. J. Prognostics Health Manag. 1(1) (2010) 1-20.

[21] X. S. Si, C. H. Hu, X. Y. Kang, D. H. Zhou, A residual storage life prediction approach for systems with operation state swithches, IEEE Trans. Ind. Electron. 61(11) (2014) 6304-6315.

[22] X. S. Si, W. B. Wang, M. Y. Chen, C. H. Hu, D. H. Zhou, A degradation path-dependent approach for remaining useful life estimation with an exact yet closed-form solution, Eur. J. Oper. Res. 226(1) (2013) 53-66.

[23] X. S. Si, W. B. Wang, C. H. Hu, M. Y. Chen, D. H. Zhou, A Wiener process-based degradation model with a recursive filter algorithm for remaining useful life estimation, Mech. Syst. Signal Process. 35(1-2) (2013) 219-237.

[24] X. S. Si, W. B. Wang, C. H. Hu, D. H. Zhou, Remaining useful life estimation-a review on the statistical data driven approaches, Eur. J. Oper. Res. 213(1) (2011) 1-14.

[25] X. S. Si, W. B. Wang, C. H. Hu, D. H. Zhou, Estimating remaining useful life with three-source variability in degradation modeling, IEEE Trans. Rel. 63(1) (2014) 167-190.

[26] T. B. Schon, A. Wills, B. Ninness, System identification of nonlinear state-space models, Automatica 47(1) (2011) $39-49$.

[27] X. S. Si, D. H. Zhou, A generalized result for degradation model based reliability estimation, IEEE Trans. Autom. Sci. Eng. 11(2) (2014) 632-637.

[28] J. M. Van Noortwijk, A survey of the application of Gamma processes in maintenance, Reliab. Eng. Syst. Saf. 94(1) (2009) 2-21.

[29] X. Wang, Wiener processes with random effects for degradation data, J. Multivariate Anal. 101(2) (2010) $340-351$.

[30] X. Wang, D. Xu, An inverse gaussian process model for degradation data, Technometrics 52(2) (2010) $188-197$.

[31] Z. S. Ye, N. Chen, The inverse Gaussian process as a degradation model, Technometrics 56 (2014) 302-311.

[32] Z. S. Ye, Y. Wang, K.-L. Tsui, M. Pecht, Degradation data analysis using Wiener processes with measurement errors, IEEE Trans. Rel. 62(4) (2013) 772-780.

[33] Z. S. Ye, M. Xie, L.C. Tang, N. Chen, Semiparametric estimation of Gamma processes for deteriorating products, Technometrics 56(4) (2014) 504-513.

[34] E. Zio, F. D. Maio, Fatigue crack growth estimation by relevance vector machine, Expert Syst. Appl. 39(12) (2012) 10681-10692.

[35] R.S. Zhou, N. Serban, N. Gebraeel, Degradation modeling applied to residual lifetime prediction using functional data analysis, Ann. Appl. Stat. 5(2B) (2011) 1586-1610 


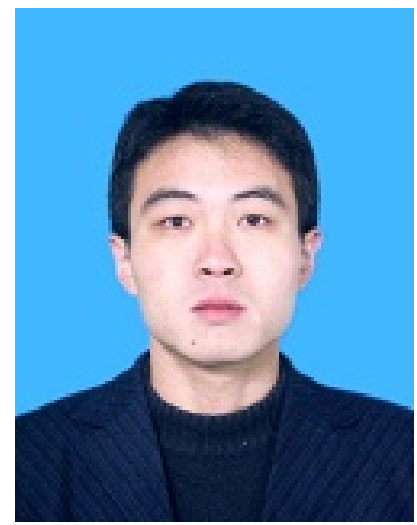

Donghui Pan received the M.S. degree in computational mathematics from Anhui University, Hefei, China, in 2010, and the Ph.D. degrees in control science and engineering from Huazhong University of Science and Technology, Wuhan, China, in 2014. He is currently with the School of Mathematical Sciences, Anhui University. His current research interests include fault diagnostics and prognostics, degradation modeling, reliability analysis of complex systems, machine learning and pattern recognition.

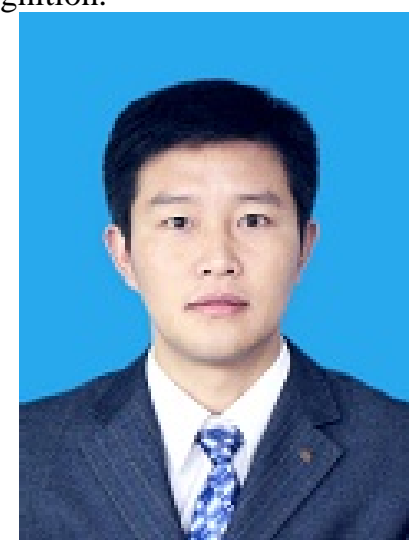

Jia-Bao Liu received the B.S. degree in mathematics and applied mathematics from Wanxi University, Anhui, China, in 2005, and the M.S. degree in mathematics and applied mathematics from Anhui University, Anhui, China, 2009, respectively. He was a visiting researcher with Southeast University, Nanjing, China, in 2013. He is currently an associate professor, and is working toward the Ph.D. degree at Anhui University, Hefei, China. His current research interests include fractional calculus theory, graph theory and its applications, neural networks, and complex dynamical networks.

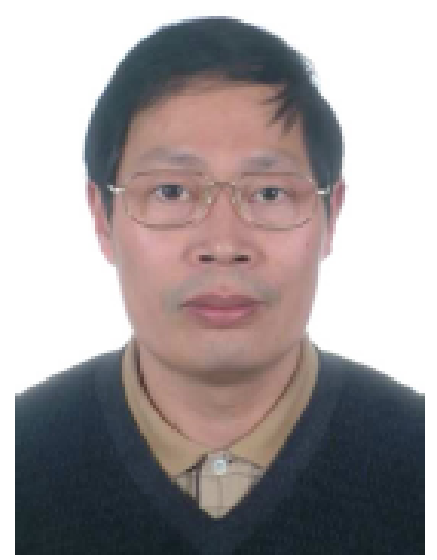

Jinde Cao (M'07-SM'07-F'16) Jinde Cao received the B.S. degree from Anhui Normal University, Wuhu, China, the M.S. degree from Yunnan University, Kunming, China, and the Ph.D. degree from Sichuan University, Chengdu, 
China, all in mathematics/applied mathematics, in 1986, 1989 and 1998, respectively. From March 1989 to May 2000 , he was with the Yunnan University. In May 2000, he joined the Department of Mathematics, Southeast University, Nanjing, China. From July 2001 to June 2002, he was a Postdoctoral Research Fellow at the Department of Automation and Computer-Aided Engineering, Chinese University of Hong Kong, Hong Kong. In the period from 2006 to 2008, he was a Visiting Research Fellow and a Visiting Professor at the School of Information Systems, Computing and Mathematics, Brunel University, UK. On August 2014, he was a Visiting Professor at the School of Electrical and Computer Engineering, RMIT University, Australia. Currently, he is a distinguished professor and doctoral advisor at the Southeast University, prior to which he was a Professor at Yunnan University from 1996 to 2000. He is the author or coauthor of more than 400 journal papers and five edited books. His research interests include nonlinear systems, neural networks, complex systems, complex networks, stability theory, and applied mathematics. Dr. Cao was an Associate Editor of the IEEE Transactions on Neural Networks, Journal of the Franklin Institute and Neurocomputing. $\mathrm{He}$ is an Associate Editor of the IEEE Transactions on Cybernetics, Differential Equations and Dynamical Systems, Mathematics and Computers in Simulation, and Neural Networks. Dr. Cao is a Fellow of IEEE, and also a Reviewer of Mathematical Reviews and Zentralblatt-Math. He is a ISI Highly-Cited Researcher in Mathematics, Computer Science and Engineering listed by Thomson Reuters. 
Donghui Pan received the M.S. degree in computational mathematics from Anhui University, Hefei, China, in 2010, and the Ph.D. degrees in control science and engineering from Huazhong University of Science and Technology, Wuhan, China, in 2014. He is currently with the School of Mathematical Sciences, Anhui University. His current research interests include fault diagnostics and prognostics, degradation modeling, reliability analysis of complex systems, machine learning and pattern recognition.

Jia-Bao Liu received the B.S. degree in mathematics and applied mathematics from Wanxi University, Anhui, China, in 2005, and the M.S. degree in mathematics and applied mathematics from Anhui University, Anhui, China, 2009, respectively. He was a visiting researcher with Southeast University, Nanjing, China, in 2013. He is currently a associate professor, and is working toward the PH.D. degree at Anhui University, Hefei, China. His current research interests include fractional calculus theory, graph theory and its applications, neural networks, and complex dynamical networks.

Jinde Cao (M'07-SM'07-F'16) received the B.S. degree from Anhui Normal University, Wuhu, China, the M.S. degree from Yunnan University, Kunming, China, and the Ph.D. degree from Sichuan University, Chengdu, China, all in mathematics/applied mathematics, in 1986, 1989 and 1998, respectively. From March 1989 to May 2000, he was with the Yunnan University. In May 2000, he joined the Department of Mathematics, Southeast University, Nanjing, China. From July 2001 to June 2002, he was a Postdoctoral Research Fellow at the Department of Automation and Computer-Aided Engineering, Chinese University of Hong Kong, Hong Kong. In the period from 2006 to 2008, he was a Visiting Research Fellow and a Visiting Professor at the School of Information Systems, Computing and Mathematics, Brunel University, UK. On August 2014, he was a Visiting Professor at the School of Electrical and Computer Engineering, RMIT University, Australia. Currently, he is a distinguished professor and doctoral advisor at the Southeast University, prior to which he was a Professor at Yunnan University from 1996 to 2000. He is the author or coauthor of more than 400 journal papers and five edited books. His research interests include nonlinear systems, neural networks, complex systems, complex networks, stability theory, and applied mathematics. Dr. Cao was an Associate Editor of the IEEE Transactions on Neural Networks, Journal of the Franklin Institute and Neurocomputing. He is an Associate Editor of the IEEE Transactions on Cybernetics, Differential Equations and Dynamical Systems, Mathematics and Computers in Simulation, and Neural Networks. Dr. Cao is a Fellow of IEEE, and also a Reviewer of Mathematical Reviews and Zentralblatt-Math. He is a ISI Highly-Cited Researcher in Mathematics, Computer Science and Engineering listed by Thomson Reuters. 


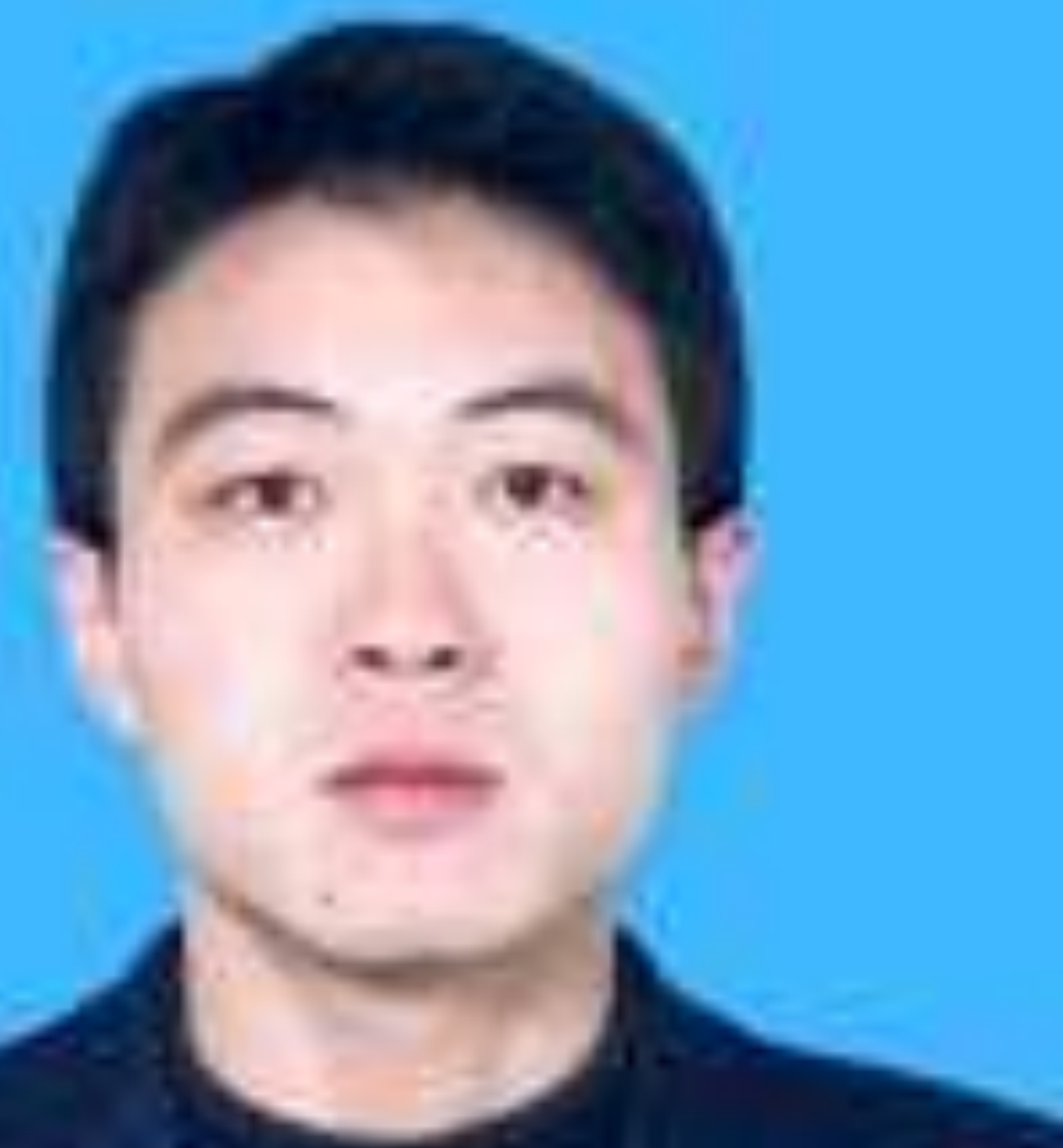




\section{Photo of the author(s) \\ Click here to download Photo of the author(s): author2.eps}

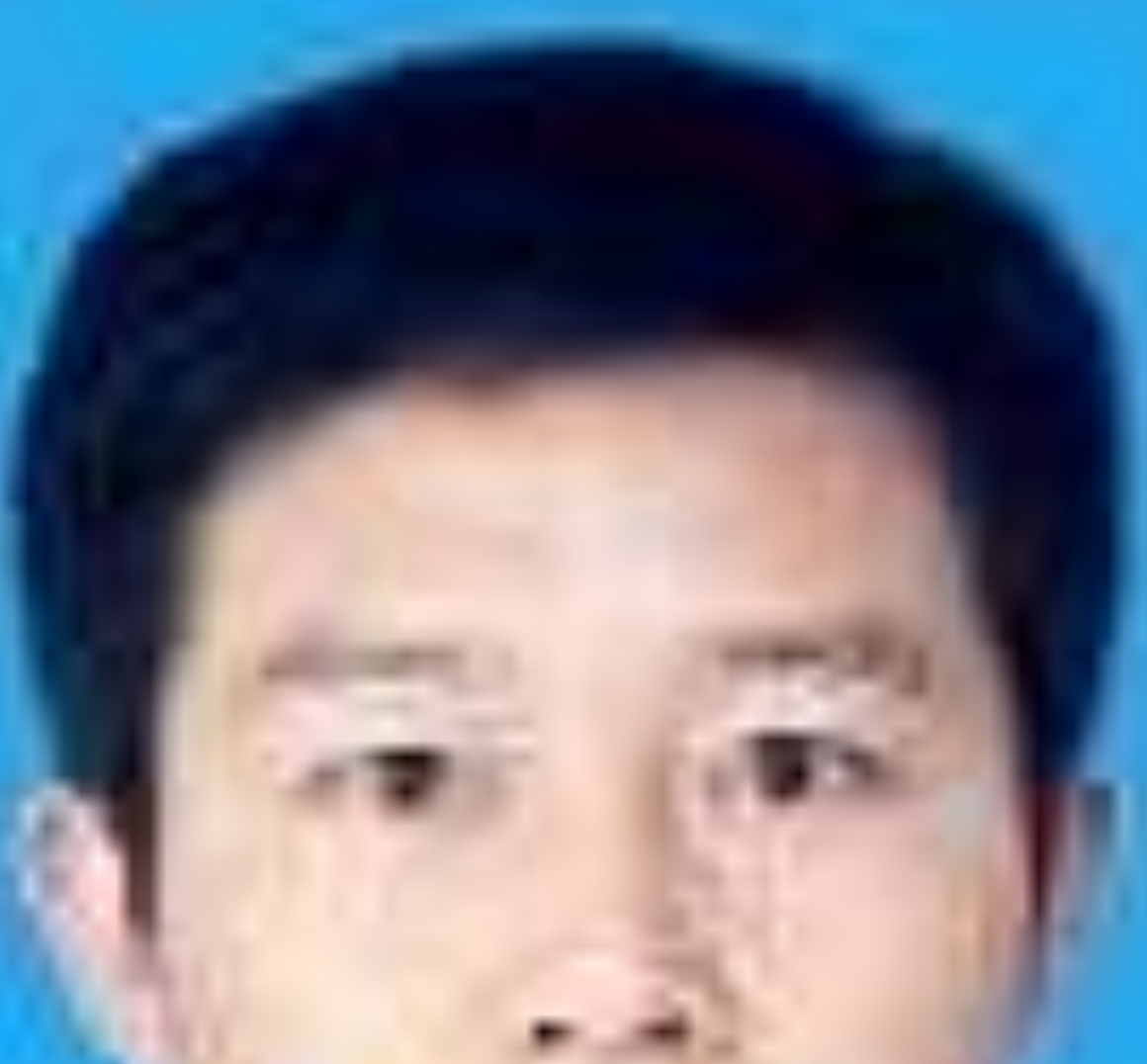


${ }^{*}$ Photo of the author(s)

\section{Click here to download Photo of the author(s): author3.eps}

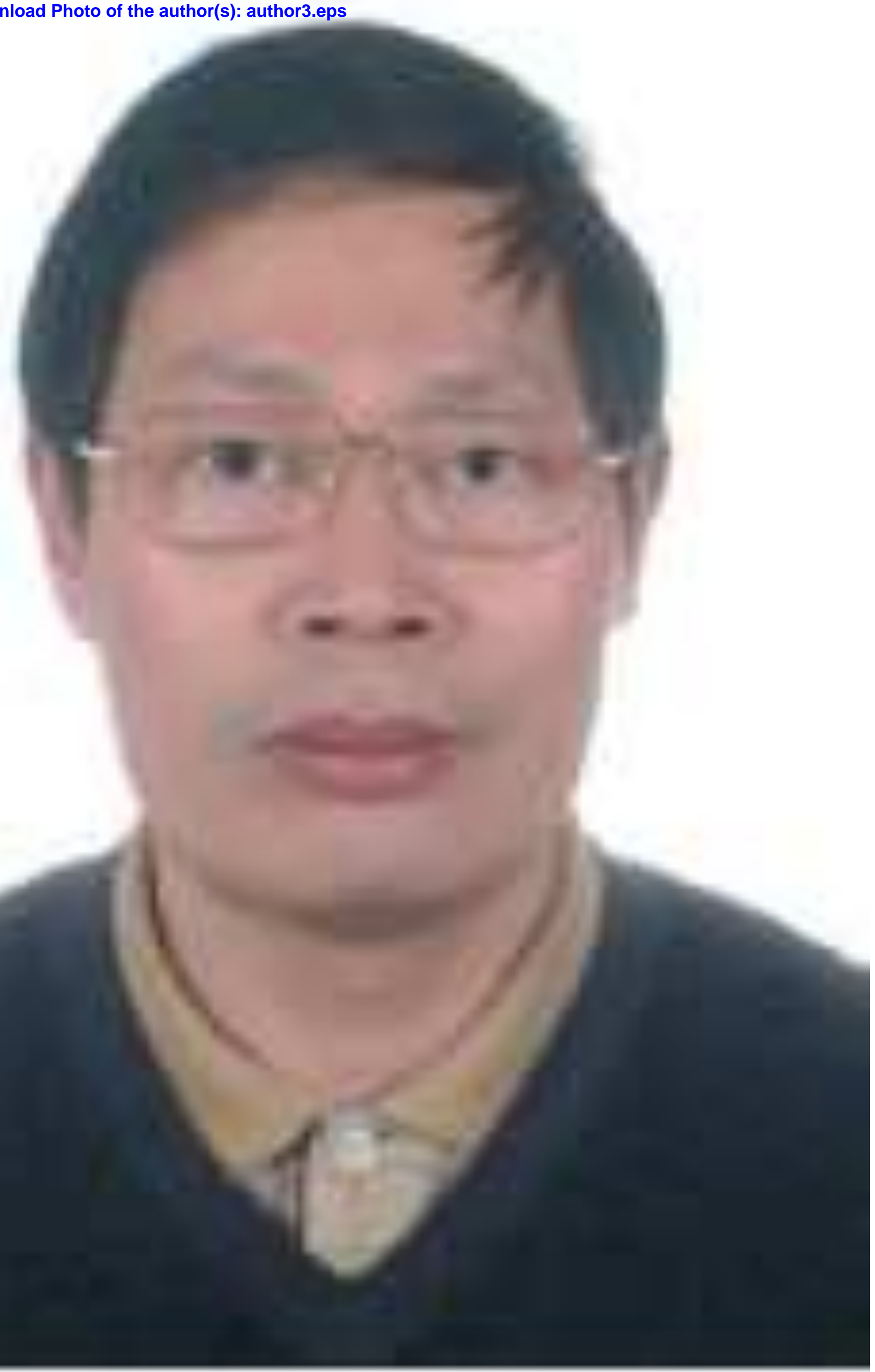


*Source Files - Latex or Word
Click here to download Source

Click here to download Source Files - Latex or Word: NEUCOM-D-15-02223R2.zip 